\title{
BMJ Open Postpartum lifestyle interventions among women with pre-eclampsia: a scoping review protocol
}

\author{
Hege Selnes Haugdahl, ${ }^{1}$ Heidi Linn Sandsæter, ${ }^{1}$ Marianne Lysne, ${ }^{1}$ \\ Ottar Bjerkeset, ${ }^{2,3}$ Lisbeth Uhrenfeldt, ${ }^{4}$ Julie Horn (D) ${ }^{5}$
}

To cite: Haugdahl HS, Sandsæter HL, Lysne M, et al. Postpartum lifestyle interventions among women with pre-eclampsia: a scoping review protocol. BMJ Open 2020;10:e035507. doi:10.1136/ bmjopen-2019-035507

- Prepublication history and additional material for this paper are available online. To view these files, please visit the journal online (http://dx.doi. org/10.1136/bmjopen-2019035507).

Received 04 November 2019 Revised 04 February 2020 Accepted 20 April 2020

\section{Check for updates}

(C) Author(s) (or their employer(s)) 2020. Re-use permitted under CC BY-NC. No commercial re-use. See rights and permissions. Published by BMJ.

${ }^{1}$ Nord-Trøndelag Hospital Trust, Levanger, Norway

${ }^{2}$ Faculty of Nursing and Health Sciences, Nord University,

Levanger, Norway

${ }^{3}$ Department of Mental Health, Faculty of Medicine and Health Sciences, Norwegian University of Science and Technology, Trondheim, Norway

${ }^{4}$ Nord University, Bodø, Norway

${ }^{5}$ HUNT Research Centre,

Department of Public Health and Nursing, Norwegian University of Science and Technology, Levanger, Norway

Correspondence to

Dr Julie Horn;

julie.horn@ntnu.no

\section{ABSTRACT}

Introduction Compared to women with normotensive pregnancies, women with a history of pre-eclampsia have a roughly fourfold increased risk of developing chronic arterial hypertension and a twofold increased risk of developing cardiovascular disease (CVD). Lifestyle changes, such as increased physical activity, weight loss, smoking cessation and healthy diet, are effective for CVD prevention in the general population. However, no scoping review or systematic review of postpartum lifestyle interventions among women with pre-eclampsia have, to our best knowledge, been performed. The objective of this scoping review is to provide an overview of the available research literature on postpartum lifestyle interventions to reduce the risk of CVD among women with pre-eclampsia. Methods and analysis The protocol is based on the framework outlined by Arksey and 0'Malley. Databases to be searched include: PubMed, Embase CINAHL and the JBI Database of Systematic Reviews and Implementation Reports. The search will be performed after the publication of this protocol (estimated to be 1 June 2020) and will be repeated 1 month prior to the submission for publication of the final review (estimated to be 1 January 2021). The review will consider studies that include women in the postpartum period (in particular, but not restricted to, the first 12 months after delivery), with a history of pre-eclampsia. Data will be extracted by two independent reviewers using a data extraction tool including specific details about the population, concept, context, study methods and key findings relevant to the review objective. Any disagreements between the reviewers will be resolved through discussion, or with a third reviewer. The extracted data will be presented in diagrammatic or tabular form that align with the objective of this scoping review. A narrative summary will accompany the tabulated and/or charted results and will describe how the results relate to the reviews objective and questions.

Ethics and dissemination Since all data will be obtained from publicly available materials, the proposed scoping review does not require ethical approval. The results will be submitted for publication in an open-access peerreviewed journal and presented at relevant conferences.

\section{INTRODUCTION}

\section{Background}

In the wake of growing awareness of cardiovascular disease (CVD) as the leading cause of death among women, female-specific risk

\section{Strengths and limitations of this study}

This will be the first scoping review to provide an overview of the available research literature on postpartum lifestyle interventions among women with pre-eclampsia.

- Results from this scoping review will be used to further the understanding of the breadth of current postpartum interventions and to identify current gaps in knowledge.

- Studies published in Danish, English, French, German, Norwegian, Spanish and Swedish will be considered for inclusion in this review.

- The size of the effects of postpartum lifestyle intervention on cardiovascular risk factors or risk of cardiovascular disease are outside the scope of the proposed review.

factor have been included in CVD prevention guidelines. ${ }^{1}$ Pre-eclampsia, characterised by new onset of arterial hypertension in pregnancy accompanied by proteinuria or/and organ dysfunction, affects approximately $5 \%-8 \%$ of all pregnancies globally, ${ }^{2}$ with higher risk among first time pregnancies. ${ }^{3}$ Severe pre-eclampsia, defined by severe hypertension or with signs of end organ failure, ${ }^{4}$ can progress rapidly and is associated with increased maternal and perinatal morbidity and mortality. ${ }^{5}$ Compared with women with normotensive pregnancies, women with a history of pre-eclampsia have a roughly fourfold increased risk of developing chronic hypertension and a twofold increased risk of CVD. ${ }^{6}$ Several studies have shown that the severity of pre-eclampsia is positively associated with risk of future CVD and reported a fourfold to sevenfold increased risk of CVD among women who experienced severe pre-eclampsia. ${ }^{7}$ Furthermore, women with a history of severe pre-eclampsia are more likely to, directly related to their pre-eclampsia, experience post-traumatic stress disorder (PTSD) or PTSD symptoms ${ }^{8-10}$ which in turn are associated with increased risk of CVD. ${ }^{11}$ 
Lifestyle changes such as increased physical activity, weight loss, smoking cessation and healthier diets have proven effective to prevent the development of CVD in the general population. ${ }^{12}{ }^{13}$ The potential benefits of lifestyle modifications might be even greater when initiated early in life. ${ }^{14}$ Pregnancy has been described as a physiological stress test unmasking a woman's predisposition to $\mathrm{CVD}^{15}$ and represents an opportunity for prevention efforts. ${ }^{16}$ Pregnancy and the postpartum period, defined here as up to 12 months after delivery, is a period when women usually are in close contact with the healthcare system and might be more responsive to suggestions to change their lifestyle. ${ }^{17}$ Being responsible for a baby may also be a motivating factor for lifestyle modification, ${ }^{18}$ which could facilitate implementing a healthier lifestyle. ${ }^{19}$ However, some recent studies have questioned the effectiveness of postpartum interventions as the effect of lifestyle interventions after pregnancy is smaller than studies of lifestyle intervention among postmenopausal women. ${ }^{121320}$

\section{Rationale}

A scoping review is suitable to summarise and disseminate research findings and to identify research gaps in existing literature. ${ }^{21}$ While a systematic review typically focuses on a well-defined question where appropriate study designs can be identified in advance, a scoping study address broader topics where many different study designs might be applicable. ${ }^{21}$ The current scoping review aims to provide an overview of the available research literature on postpartum lifestyle interventions among women with pre-eclampsia due to the following reasons: first, the postpartum period poses several potential barriers to lifestyle modifications including caregiving duties, lack of time and fatigue. ${ }^{182-24}$ Second, knowledge of the relationship between pre-eclampsia and increased CVD risk is pivotal to advise and implement lifestyle changes. However, previous studies in the USA have shown that women with pre-eclampsia were unfamiliar with the relationship between pre-eclampsia and increased future CVD risk. ${ }^{22}$ Pre-eclampsia was rather perceived as an isolated event in pregnancy without any impact on future health, ${ }^{22}$ which emphasise the need for interventions, empowerment and patient involvement. Third, postpartum intervention studies are more commonly directed at gestational diabetes, a pregnancy complication similarly tied to an increased risk of CVD. A systematic review on this topic, reported poor recruitment rates in most studies, underscoring the lack of evidence of how to engage women and improve health in this population. ${ }^{24}$ Smith et al suggested adopting a more salutogenically focused approach to maternity care, emphasising a health promotion perspective instead of solely focusing on risk for diseases. ${ }^{25}$

Finally, changing lifestyle is challenging and difficult for most people, ${ }^{26}$ due to psychological distress and previous negative experiences in achieving lifestyle changes. ${ }^{27}$ Further, women with a history of severe preeclampsia might face additional postpartum challenges related to obstetric emergencies and neonatal morbidity. ${ }^{9}$ Currently, there is a limited overview of whether postpartum interventions among women with severe preeclampsia include the specific challenges these women have to face.

A preliminary search in MEDLINE via PubMed on the area of 'pre-eclampsia', 'postpartum period', 'lifestyle interventions' and 'cardiovascular diseases' was performed in September 2019. No scoping review or systematic review of postpartum lifestyle interventions among women with pre-eclampsia were found. Additional studies are needed to further tailor the delivery of postpartum health promotion lifestyle interventions. ${ }^{20}$

\section{Objectives}

The objective of this scoping review is to provide an overview over existing evidence of postpartum lifestyle interventions to reduce CVD risk among women with preeclampsia. Due to the association of severe pre-eclampsia and PTSD with CVD, we will also search for interventions addressing PTSD to identify interventions to this specific group of women. Results from this scoping review will be used to identify the breadth of postpartum interventions for women with a recent history of pre-eclampsia and current gaps in knowledge. This may inform future research directions and a systematic review aiming to evaluate the benefit of lifestyle interventions to reduce CVD risk among women with pre-eclampsia.

\section{METHODS AND ANALYSIS}

The proposed scoping review will broadly follow a framework guided by Arksey and O'Malley ${ }^{21}$ and further developed by Levac et $a l^{28}$ and the Joanna Briggs Institute methodology for scoping reviews. ${ }^{29}$ Our methods will comprise five major steps: (1) identifying the research questions, (2) identifying relevant studies, (3) study selection, (4) charting the data and (5) collating, summarising and reporting the results. ${ }^{21}$ The Preferred Reporting Items for Systematic Reviews and Meta-Analyses (PRISMA)Extension for Scoping Reviews: checklist and explanation will be followed. ${ }^{30}$

\section{Stage 1: identifying the research questions}

We have identified one overarching research question to guide the systematic search strategy: "what is known about postpartum lifestyle interventions to reduce CVD risk among women with pre-eclampsia?'. This question should allow us to adequately capture the breadth of existing literature, while providing the opportunity for guiding research questions to be added or modified as required throughout the course of the study. During the preliminary exploratory review, we have identified several secondary questions to guide the subsequent stages of the scoping review, and to complement the overarching question above. These questions will include, but will not be limited to: 
Table 1 PCC grid showing identified search terms with truncated keyword and MeSH terms for PubMed

\begin{tabular}{llll} 
& Population & Concept & Context \\
\hline Definition & $\begin{array}{l}\text { Women in the postpartum } \\
\text { period defined here as up to } \\
12 \text { months after delivery with a } \\
\text { history of pre-eclampsia }\end{array}$ & $\begin{array}{l}\text { Postpartum lifestyle } \\
\text { interventions }\end{array}$ & $\begin{array}{l}\text { Postpartum healthcare } \\
\text { settings, including hospital } \\
\text { care, community care, home } \\
\text { care settings }\end{array}$ \\
& $\begin{array}{ll}\text { Patients } \\
\text { PubMed (MeSH-terms) }\end{array}$ & Lifestyle & Tertiary healthcare \\
& Pre-eclampsia & Healthy lifestyle & Hospitals, public \\
& Postnatal care & Patient education & Community health services \\
& $\begin{array}{l}\text { Hypertension, pregnancy- } \\
\text { induced }\end{array}$ & & Hospital care \\
& & Lifestyle intervention* & Community care \\
Text words & & Home care settings
\end{tabular}

*Identifies search term with truncated keyword.

$\mathrm{MeSH}$, medical subject headings; PCC, population concept context.

1. What types of postpartum lifestyle interventions among women with pre-eclampsia have been described?

- Do the studies focus on (a) the identification of risk factors and reduction of adverse outcomes or (b) salutogenic approach?

- Are some of the studies tailored to specific subgroups of women with pre-eclampsia (severe preeclampsia or women at higher risk of PTSD)?

2. When is the recommended time of recruitment for postpartum lifestyle interventions?

3. What evidence exists of the effectiveness of the postpartum lifestyle interventions?

4. How have women's perceptions of the interventions been reported?

\section{Stage 2: identifying relevant studies}

The initial search strategy (online supplementary appendix 1) was developed in collaboration with a medical librarian (ML). Databases to be searched as part of this review include: PubMed, Embase, the Cochrane Central Register of Controlled Trials, CINAHL and the JBI Database of Systematic Reviews and Implementation Reports. Literature search strategies will be developed using medical subject headings (MeSH-terms) and text words related to the population, concept and context of the study as shown in table 1 .

Empirical studies with either qualitative or quantitative data published in Danish, English, French, German, Norwegian, Spanish and Swedish will be considered for inclusion in this review. The scoping review will exclude all types of reviews, protocols, book chapters, editorial letters and guidelines. Qualitative results from other languages than English will be translated into English and results in the original language will be presented in brackets.

A three-step search strategy will be used. An initial limited search of PubMed, Embase and CINAHL will be undertaken, followed by analysis of the text words contained in the title and abstract, and of the index terms used to describe the article. A second search using all identified keywords and index terms will then be undertaken across all included databases. Third, the reference list of all studies will be screened for additional studies. We will consider studies published from 2000 onwards.

\section{Stage 3: selecting studies}

Following the search, all identified citations will be uploaded into the Rayyan QCRI systematic review app and duplicates will be removed. ${ }^{31}$ The review process will consist of two levels. Each level will be performed by two data reviewers who will independently consider the studies against inclusion and exclusion criteria. The first screening process will include reading the titles and abstracts to reach the following decisions: (1) if at least one reviewer agrees to include or consider the abstract or title to be inconclusive, the study will be moved to the second level of screening; (2) for any study both reviewers agree to exclude, the study will be excluded. All studies at this level will be recorded by both reviewers using the RAYYAN systematic review app.

In the second level of the screening process, the full text will be assessed by two independent reviewers. Reasons for exclusion of studies will be recorded and reported in the scoping review. Any disagreements between the reviewers will be resolved through discussion, or with a third reviewer. The results of the search and study selection will be reported in a PRISMA flow diagram. ${ }^{30}$

The following inclusion criteria will be met: (1) studies that include women in the postpartum period (in particular, but not restricted to, the first 12 months after delivery) with a history of pre-eclampsia (severe or non-severe) and (2) any intervention to reduce women's elevated CVD risk, including but not limited to lifestyle modifications such as increased physical activity, healthy eating and smoking cessation. Based on the link between 
pre-eclampsia and PTSD, we will also include interventions to promote mental health and well-being. Furthermore, we will review how women's perceptions of the lifestyle interventions after child birth (facilitators and barriers) have been reported.

\section{Stage 4: charting the data}

Data from the included studies will be collected and charted by two reviewers using a data extraction tool. The data extracted will include specific details about the population, concept, context, study methods and key findings relevant to the review objective. A draft charting table is provided (online supplementary appendix 2). As a preliminary step, the reviewers will independently extract data from the first five studies using the data-charting table and meet to determine whether the approach to data extraction is consistent with the purpose of the study. ${ }^{28}$ The draft data extraction tool will be modified and revised as necessary during the process of extracting data from each included study. Modifications will be detailed in the full scoping review article. Any disagreements that arise between the reviewers will be resolved through discussion, or with a third reviewer.

\section{Stage 5: collating, summarising and reporting the results}

The extracted data in stage 4 of the proposed scoping review will be collated, summarised and reported in a manner that aligns with the objective of this scoping review (online supplementary appendix 3). Tabular and graphical representations of the data may be used to illustrate the identified results, and will be supported by narrative descriptions of data. The meaning of the findings and implications for future research and practice will be discussed. ${ }^{28}$

\section{Patient and public involvement}

Patients and public were not involved in the design and conception of this study.

\section{ETHICS AND DISSEMINATION}

This proposed scoping review does not require ethical approval as data will be collected through the review of published peer-reviewed literature and grey literature. This scoping review will provide a comprehensive overview of existing evidence of the field, which will help to guide postpartum lifestyle interventions to reduce CVD risk among women with pre-eclampsia. The results will be submitted for publication in an open-access peerreviewed journal and presented at relevant conferences.

Acknowledgements We thank Nord University, Faculty of Nursing and Health Sciences who provided first author with training in scoping reviews and, librarian Marit Veie, who advised on search strategy.

Contributors HSH, HLS, OB and JH jointly conceived the study, outlined the protocol and developed the research questions. HSH, HLS, LU, JH and ML contributed to the development of the search strategy and development of the systematic search framework. HSH and JH drafted the manuscript. All authors further revised the manuscript and approved the final text.
Funding This review was supported by a grant from the Liaison Committee for education, research and innovation in Central Norway.

Competing interests None declared.

Patient consent for publication Not required.

Provenance and peer review Not commissioned; externally peer reviewed.

Open access This is an open access article distributed in accordance with the Creative Commons Attribution Non Commercial (CC BY-NC 4.0) license, which permits others to distribute, remix, adapt, build upon this work non-commercially, and license their derivative works on different terms, provided the original work is properly cited, appropriate credit is given, any changes made indicated, and the use is non-commercial. See: http://creativecommons.org/licenses/by-nc/4.0/.

ORCID iD

Julie Horn http://orcid.org/0000-0003-1344-9707

\section{REFERENCES}

1 Mosca L, Benjamin EJ, Berra K, et al. Effectiveness-based guidelines for the prevention of cardiovascular disease in women--2011 update: a guideline from the American Heart Association. J Am Coll Cardiol 2011;57:1404-23.

2 Abalos E, Cuesta C, Grosso AL, et al. Global and regional estimates of preeclampsia and eclampsia: a systematic review. Eur J Obstet Gynecol Reprod Biol 2013;170:1-7.

3 Klungsøyr K, Morken NH, Irgens L, et al. Secular trends in the epidemiology of pre-eclampsia throughout 40 years in Norway: prevalence, risk factors and perinatal survival. Paediatr Perinat Epidemiol 2012;26:190-8.

4 Staff ACA, Andersgaard AB, Henriksen T, et al. Chapter 28 hypertensive disorders of pregnancy and eclampsia. Eur J Obstet Gynecol Reprod Biol 2016;201:171-8.

5 Gifford RW, August PA, Cunningham G, et al. Report of the National high blood pressure education program Working group on high blood pressure in pregnancy 2000.

6 Bellamy L, Casas J-P, Hingorani AD, et al. Pre-Eclampsia and risk of cardiovascular disease and cancer in later life: systematic review and meta-analysis. BMJ 2007;335:974.

7 McDonald SD, Malinowski A, Zhou Q, et al. Cardiovascular sequelae of preeclampsia/eclampsia: a systematic review and meta-analyses. Am Heart J 2008;156:918-30.

8 Hoedjes M, Berks D, Vogel I, et al. Symptoms of post-traumatic stress after preeclampsia. J Psychosom Obstet Gynaecol 2011;32:126-34.

9 Bastos MH, Furuta M, Small R, et al. Debriefing interventions for the prevention of psychological trauma in women following childbirth. Cochrane Database Syst Rev 2015;4:Cd007194.

10 Furuta M, Sandall J, Bick D. A systematic review of the relationship between severe maternal morbidity and post-traumatic stress disorder. BMC Pregnancy Childbirth 2012;12:125.

11 Edmondson D, Kronish IM, Shaffer JA, et al. Posttraumatic stress disorder and risk for coronary heart disease: a meta-analytic review. Am Heart J 2013;166:806-14.

12 Liu K, Daviglus ML, Loria CM, et al. Healthy lifestyle through young adulthood and the presence of low cardiovascular disease risk profile in middle age: the coronary artery risk development in (young) adults (cardia) study. Circulation 2012;125:996-1004.

13 Stevens JW, Khunti K, Harvey R, et al. Preventing the progression to type 2 diabetes mellitus in adults at high risk: a systematic review and network meta-analysis of lifestyle, pharmacological and surgical interventions. Diabetes Res Clin Pract 2015;107:320-31.

14 Weintraub WS, Daniels SR, Burke LE, et al. Value of primordial and primary prevention for cardiovascular disease: a policy statement from the American heart association. Circulation 2011;124:967-90.

15 Sattar N. Do pregnancy complications and CVD share common antecedents? Atheroscler Supp/ 2004;5:3-7.

16 Smith GN, Louis JM, Saade GR. Pregnancy and the postpartum period as an opportunity for cardiovascular risk identification and management. Obstet Gynecol 2019;134:851-62.

17 Nascimento SL, Pudwell J, Surita FG, et al. The effect of physical exercise strategies on weight loss in postpartum women: a systematic review and meta-analysis. Int J Obes 2014;38:626-35.

18 Edvardsson K, Ivarsson A, Eurenius E, et al. Giving offspring a healthy start: parents' experiences of health promotion and lifestyle change during pregnancy and early parenthood. BMC Public Health 2011;11:936. 
19 McBride CM, Emmons KM, Lipkus IM. Understanding the potential of teachable moments: the case of smoking cessation. Health Educ Res 2003;18:156-70.

20 Goveia P, Cañon-Montañez W, Santos DdeP, et al. Lifestyle intervention for the prevention of diabetes in women with previous gestational diabetes mellitus: a systematic review and meta-analysis. Front Endocrinol 2018;9:583.

21 Arksey H, O'Malley L. Scoping studies: towards a methodological framework. Int J Soc Res Methodol 2005;8:19-32.

22 Seely EW, Rich-Edwards J, Lui J, et al. Risk of future cardiovascular disease in women with prior preeclampsia: a focus group study. BMC Pregnancy Childbirth 2013;13:240.

23 Sandsæter HL, Horn J, Rich-Edwards JW, et al. Preeclampsia, gestational diabetes and later risk of cardiovascular disease: Women's experiences and motivation for lifestyle changes explored in focus group interviews. BMC Pregnancy Childbirth 2019;19.

24 Jones EJ, Fraley HE, Mazzawi J. Appreciating recent motherhood and culture: a systematic review of multimodal postpartum lifestyle interventions to reduce diabetes risk in women with prior gestational diabetes. Matern Child Health J 2017;21:45-57.
25 Smith V, Daly D, Lundgren I, et al. Salutogenically focused outcomes in systematic reviews of intrapartum interventions: a systematic review of systematic reviews. Midwifery 2014;30:e151-6.

26 Kwasnicka D, Dombrowski SU, White M, et al. Theoretical explanations for maintenance of behaviour change: a systematic review of behaviour theories. Health Psychol Rev 2016;10:277-96.

27 Følling IS, Solbjør M, Helvik A-S. Previous experiences and emotional baggage as barriers to lifestyle change - a qualitative study of Norwegian Healthy Life Centre participants. BMC Fam Pract 2015;16:73.

28 Levac D, Colquhoun H, O'Brien KK. Scoping studies: advancing the methodology. Implement Sci 2010;5:69.

29 Peters MDJ, Godfrey CM, Khalil H, et al. Guidance for conducting systematic scoping reviews. Int J Evid Based Healthc 2015;13:141-6.

30 Tricco AC, Lillie E, Zarin W, et al. PRISMA extension for scoping reviews (PRISMA-ScR): checklist and explanation. Ann Intern Med 2018;169:467-73.

31 Ouzzani M, Hammady H, Fedorowicz Z, et al. Rayyan-a web and mobile APP for systematic reviews. Syst Rev 2016;5:210. 